
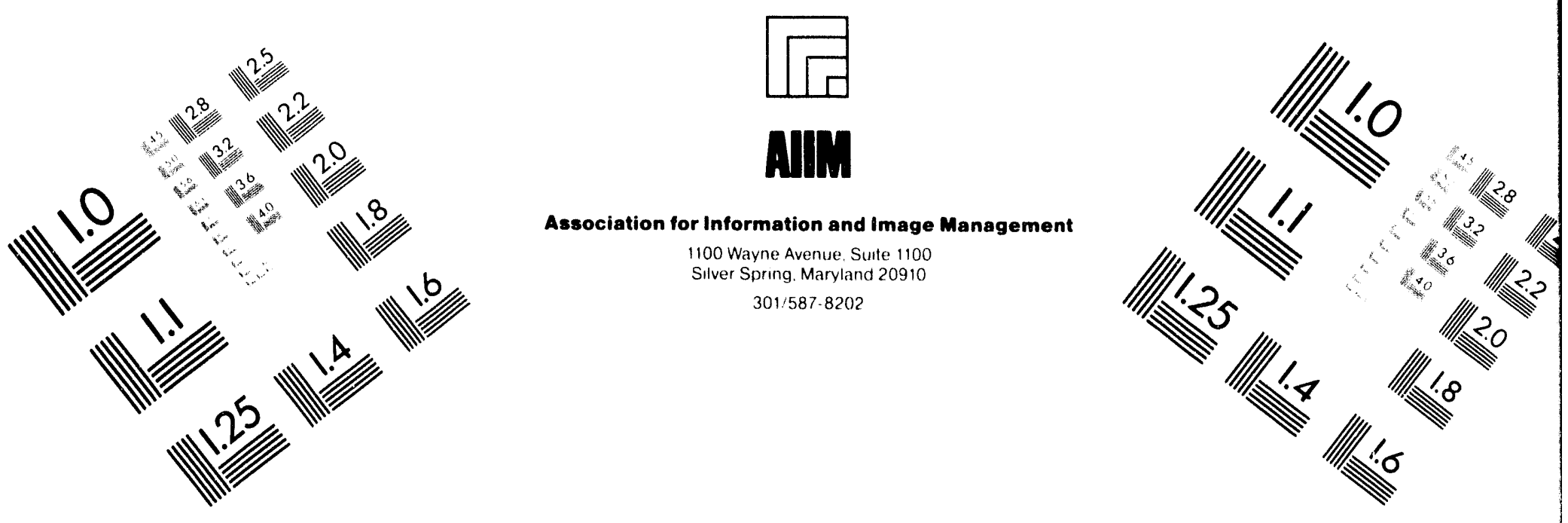

Centimeter

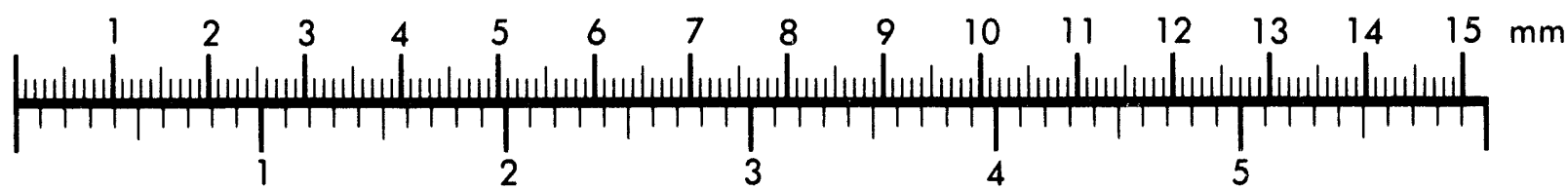

Inches
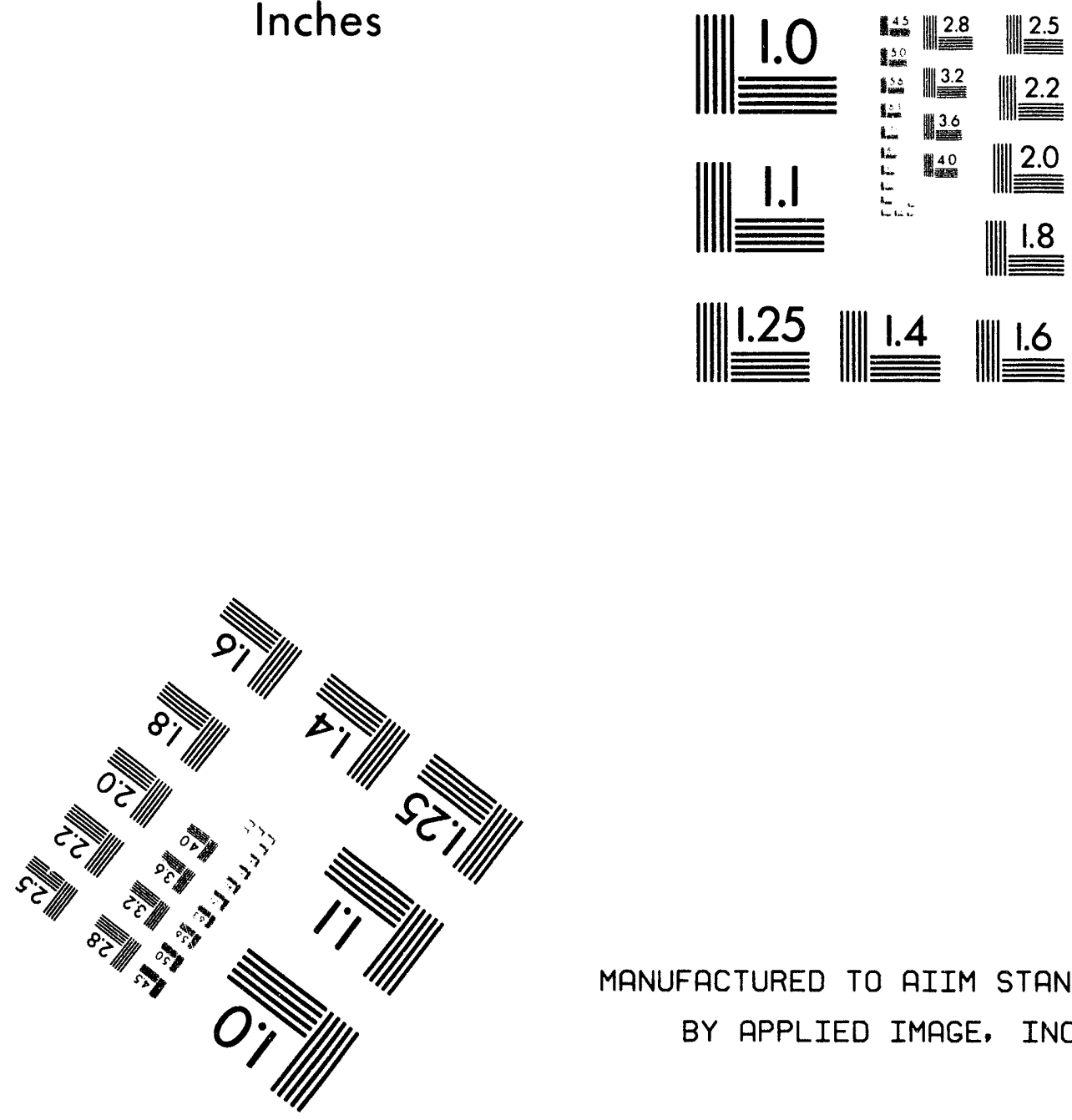

MANUFACTURED TO AIIM STANDARDS

BY APPLIED IMAGE, INC.

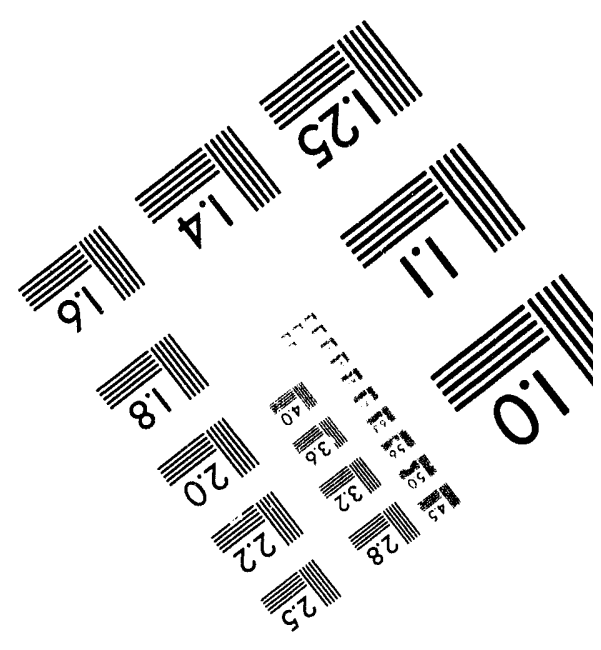



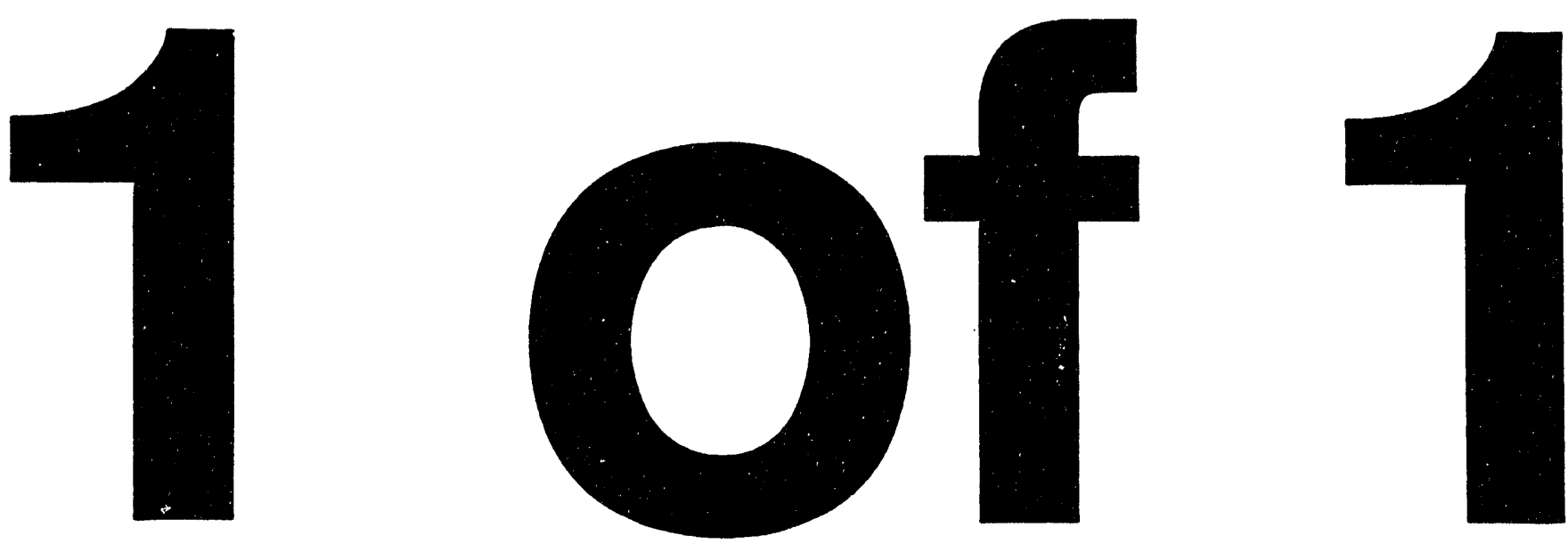
E. I. Ơu Pont de Nemour's \& Company

Explosives Department

Atom1c Erengy D1viston

W1imingten $38, D \leq 1 \equiv$ rare

\author{
DCES NOT CONTAIN SR/H--767 \\ UNCLASZ:FI:D CCI:TAOOLLED \\ NUCLEAR RiFOR:AATION
}

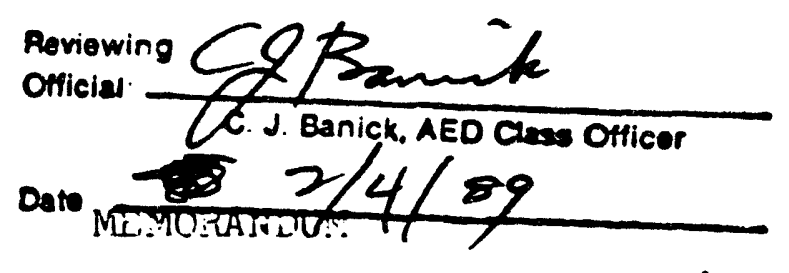

FRON: $\because$. A . FJHL
CC: R. M. Evans - B. H. Mackey

J. E. Ccle - M. H. Smith

H. worthineton

i. R. Thaje:

I. B. Tinie: - R. J. Christl

File

M. H. WahI - C.W. J. Wende

T. W. Micirs-s - W. P. Bebbingtor

i. P. jvez=sak - L. C. Feery

J. D. El-ez= - i, S. Danser

ST File

\section{ianuany 26,1956}

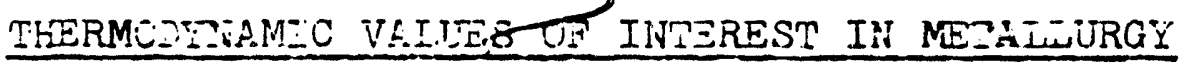

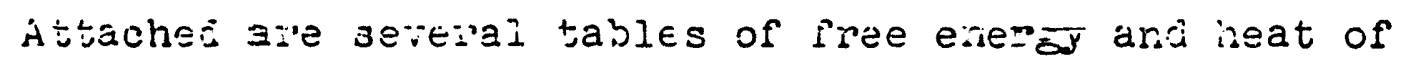
forliation for the jetals of teir eacountered in meta iung. They have. been collated mainiz from the Nos Clrcular $f^{\prime \prime} 500$, acj are on a selfconsistert basis. Fable I gives a bird's eye viei oi the reiative combinsng affinity of tine elerents, usuily in pure sclid or pure liquid states. As an be seen, the ability of one metal to replace arother depends marizedly or the conditions. The owder 1 a different for fllioriaes, criczides, oxides, sulfidss, or in silute aqueous soiution. The lastor orcer is the familiar "electanotive series", Table iI.

The vainis of the free energy or heat 0 reaction as given in Tables I jacough $V$ are for the metais an tiseir salts in the "stardard state"--1.e. Witin unit activity suci as for pure metal

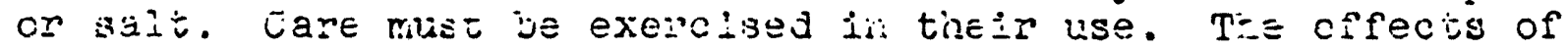
divition are in the direction as to oppose an exyested reaction's completion. For eximple, according to the table of free energies Ir the standard siates (or what is numerically nezrly the same for most cases, the reats of reaction) as shown in Tabie I, one would expect dranium to redice alliminum oxide by the reaction:

$$
\mathrm{Vic})+2 / 3 \mathrm{Ai}_{2} \mathrm{C}_{3}(\mathrm{c})=\mathrm{JO}_{2}(\mathrm{c})+4 / 3 \mathrm{Al}(\mathrm{c}) \Delta \mathrm{F}=-6 \mathrm{Kcal} / \mathrm{mol} \text { at } 298^{\circ} \mathrm{K}
$$

where the symbol (c) Incicates tise crystal or "starcard" state. Howevsr. "iranlum dissolves in alumirum and checks the completion of the reasticn. A simfiar argurnent applies, ion exampie, to the reduction of Puc? oy $\mathrm{Ca}$. This entropy effect becomes especieily large at high dilution and at elevatea temperatures. It is showr. In figures VII and VII that the dilution effect in this case is comparable to the "stancará" free energy difference at conceritrations of 5 mol percent of less at temperatines above $800^{\circ} \mathrm{K}$. Accordingly, it generally is best to ragard the stancard free energy values on ${ }^{\prime}$ as rough guldes as to whethen or not a reaction can "go". The effests of dilution and temperature upos the exten's of reaction are large, and must ustially be considered. 
The effects of dilution were calculated assuming that the solution of $U$ in $A I$ were "perfect", 1.e. that $\Delta H=0$. The calculation Sollows:

$$
\begin{aligned}
& S=\text { entropy per mol } \\
& F=\text { fre }=\epsilon \text { nergy per mol } \\
& H=r s a t o \vec{i} \text { react } \pm \text { cn per mol } \\
& \eta \text { = atsolite temcerature } \\
& R=\text { gas constant } \\
& x_{1}=\text { mol fraction of } 1 \text { th } \text { component. }
\end{aligned}
$$

(I) Since $\Delta F=\Delta \mathrm{H}-\mathrm{T} \Delta \mathrm{S}$

and in perfect sollitions

(2) $\Delta H=0, t h a n$

$$
\begin{array}{rlrl}
(\Delta \mathrm{F})_{\mathrm{T}} & =-\mathrm{T} \Delta \mathrm{S} \\
& =\text { FT } \sum \mathrm{X}_{1} \ln \mathrm{X}_{\mp} & \text { by definition } \\
(\text { cf. Dodge, Eq. IV-52) }
\end{array}
$$

in $5 \% \mathrm{U}$ in $\mathrm{AI}$

$$
\begin{aligned}
& x_{u}=\frac{w_{u} / M_{u}}{w_{u} / M_{u}+w_{A I} / M_{A I}} \\
& \text { where } W_{1}=\text { we1ght of } 1 \text { th } \text { component } \\
& M_{1}=\text { atomic weight of ith component } \\
& x_{u}=\frac{\frac{.05}{238}}{\frac{.05}{235}+\frac{.95}{27}}=.006008 \\
& \mathrm{x}_{\mathrm{A} 1}=1-\mathrm{x}_{\mathrm{u}}=.993992 \\
& \left.(\Delta \mathrm{F})_{\mathrm{T}}=! .993992 \text { In } 0.993992+.006008 \text { in } 0.006008\right) \mathrm{RT} \\
& =-.03682 \mathrm{RT} \text {. } \\
& \text { at } \mathrm{T}=1300^{\circ} \mathrm{K} \\
& \Delta \mathrm{F}_{1300}=-.03682 \times 1.986 \times 1300=-95.06 \mathrm{cal} / \mathrm{mol} \text { solution }
\end{aligned}
$$




$$
\Delta F_{1300}^{\prime}=\frac{-95.06}{.006008}=-15,820 \mathrm{cal} / \mathrm{mol} U \text { in solution }
$$

In a similar manner calculations were made for several selected temperatures and compositions.

Figure IA shows the free energy excess required to drive a reaction to the indicated level of completion if one reactant is in dilute solution.

\title{
DISCLAIMER
}

\begin{abstract}
This report was prepared as an account of work sponsored by an agency of the United States Government. Neither the United States Government nor any agency thereof, nor any of their employees, makes any warranty, express or implied, or assumes any legal liability or responsibility for the accuracy, completeness, or usefulness of any information, apparatus, product, or process disclosed, or represents that its use would not infringe privately owned rights. Reference herein to any specific commercial product, process, or service by trade name, trademark, manufacturer, or otherwise does not necessarily constitute or imply its endorsement, recommendation, or favoring by the United States Government or any agency thereof. The views and opinions of authors expressed herein do not necessarily state or reflect those of the United States Government or any agency thereof.
\end{abstract}




\section{$10^{-1}$ :

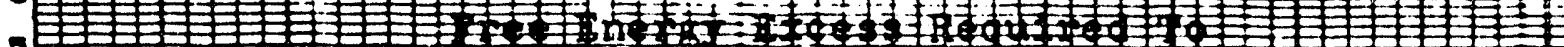

4 侓

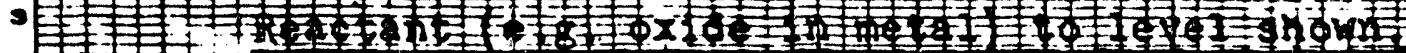

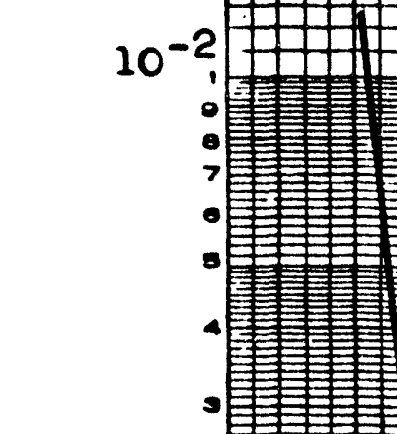

Mol

fract1on

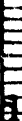

reactant

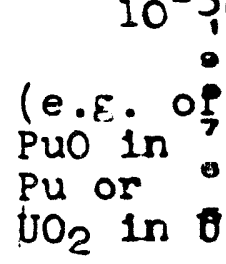

$10-3$
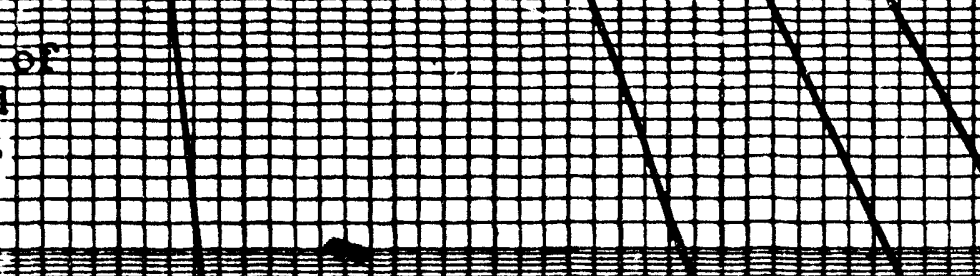


\section{TABLE I}

Heats of Formation @ $298.16^{\circ} \mathrm{K}$, Energy per Average Bond Data from NBS C1rc. 500 .

Oxide Fluoride

$\mathrm{Y}^{+++}$

$\mathrm{La}^{+++}$

$\mathrm{Ca}^{++}$

$\mathrm{Pr}^{+++}$

$\mathrm{Nd}^{+++}$

$\mathrm{Sc}^{+++}$

$\mathrm{Be}^{++}$

$\mathrm{Th}^{++++}$

$\mathrm{Mg}^{++}$

$\mathrm{Li}^{+}$

$\mathrm{Sr}^{++}$

$\mathrm{Pu}^{+++}$

$\mathrm{N}_{\mathrm{p}}^{+++}$

$\mathrm{U}^{+++}$

$\mathrm{U}^{++++}$

$\mathrm{Ba}^{++}$

$\mathrm{Al}^{+++}$

$\mathrm{Pu}+++$

$\mathrm{Ce}^{+++}$

$\mathrm{Ce}++++$

$\mathrm{Si}^{++++}$

$\mathrm{Na}^{+}$

$\mathrm{K}^{+}$

$\mathrm{Fe}^{++}$

$\mathrm{C}^{++}$
$-4 \mathrm{H}^{\circ} \mathrm{e}, \mathrm{Kcal} / \mathrm{mol}$

79 (from

76.3

Ephra1m)

75.95

74.1

73.7

73.0

73.0

71.92

71.2

70.55

$68 \pm 3$

67.5

66.7

66.52

62.8

58.3

51.4

49.7

43.2

31.9

13.208 (co,gas)
$-\Delta \mathrm{H}_{\mathrm{C}}, \mathrm{Kcal} / \mathrm{mol}$

Chloride $-\Delta \mathrm{H}^{\circ} \mathrm{f}, \mathrm{Kcal} / \mathrm{mol}$

78.3

87.9

51.1

145.2

95.0

57.7

85.9

84.8

46.97

73.6

125.7

61.2

28.0

119.3

71.2

43.7

( $\mathrm{Th}_{2} \mathrm{~S}_{3}$ )

131.8

76.7

41.5

146.3

97.70

145.2

$---$

124.9 ( $\mathrm{PuF}_{3}$, aq.)

99.0

54.1

120

76.7

73

119

71

110.8

62.8

143.5

102.78

53.0

103.7

55.4

20.27

124.9

76.7

86.8

49.8
92.5 (S1F4 gas)

136.0

134.46

$40.8\left(\mathrm{CF}_{4}, \mathrm{gas}\right)$

$\begin{array}{cc}38.3\left[\mathrm{SICl}_{4}(1 \mathrm{qq}]\right. & 17.4 \\ 97.755 & 44.6 \\ 104.175 & 50.0 \\ 40.8 & 11.36 \\ 8.3\left(\operatorname{ccl}_{4},\right. & -10.5 \\ 11 q) & \left(\operatorname{cs}_{2} 11 q\right)\end{array}$




\section{TABLE II}

\section{Pluorides}

\begin{tabular}{|c|c|c|c|c|}
\hline sompound & $\Delta \mathrm{H}_{\mathrm{r}}^{\mathrm{O}}$ & $\Delta \mathrm{H}^{0}{ }_{\mathrm{f}} / \mathrm{s}$ & $\Delta r^{0} r$ & $\Delta H^{\circ} / F$ \\
\hline L1F (c) & -145.3 & -146.3 & -139.6 & -139.6 \\
\hline $\mathrm{CaF}_{\mathrm{C}}(\mathrm{c})$ & $\because 90.3$ & -345.2 & -277.7 & -138.9 \\
\hline $\operatorname{SrF}_{2}(c)$ & $-=90.3$ & $-i+5.2$ & & \\
\hline $\operatorname{SaA}_{2}(c)$ & -286.9 & $-: 33.5$ & -274.5 & -137.3 \\
\hline$\therefore \approx F \quad(c)$ & -135.0 & $-\therefore 36.0$ & -129.3 & -129.3 \\
\hline$P(0)$ & $-13 \because .45$ & $--34 . \ddot{6}$ & -127.42 & -127.4 \\
\hline$M_{g} F_{2}(c)$ & -263.5 & $-13 i .8$ & -250.8 & -125.4 \\
\hline $\operatorname{EeP}_{2}(a q)$ & -251.4 & $-1 \geq 5.1$ & & \\
\hline $\mathrm{PuP}_{3}(\mathrm{aq})$ & $-37 \div .6$ & $-i \cong 4.9$ & & \\
\hline$\because o P_{3}(c)$ & -360 & -izc. & & \\
\hline $\mathrm{ShF}_{4}(\mathrm{c})$ & $-4 \pi T$ & -11.9 .3 & & \\
\hline$V_{3}(c)$ & -357 & -119 & -332. & -113 \\
\hline $\mathbb{N F}_{4}(c)$ & -443 & -110.8 & -421 & $-105 \cdot 3$ \\
\hline$A{ }^{\prime} F_{3}(c)$ & -311. & -203.7 & -294 & $-9 \varepsilon$. \\
\hline$=1 F_{4}(E)$ & -350 & .52 .5 & $-3=2$ & $-9 c$. \\
\hline$=54(8)$ & -162.5 & -40.8 & -151.8 & -37.95 \\
\hline$P_{2} F_{2}(a q)$ & -177.8 & -88.9 & & \\
\hline
\end{tabular}




\section{TAELE III}

\section{Chlor1des}

\begin{tabular}{|c|c|c|c|c|}
\hline compound & $\Delta \mathrm{H}_{\mathrm{f}}^{\circ}$ & $\Delta \mathrm{H}^{0}{ }_{\mathrm{f}}^{\prime \prime 心 I}$ & $\Delta \mathrm{P}^{\mathrm{C}} \mathrm{f}$ & $\Delta F^{\circ}{ }_{f} / C I$ \\
\hline$\pi \operatorname{il}(c)$ & $-20 \% .175$ & $-104 .=75$ & -97.592 & -97.592 \\
\hline$E \equiv z 2_{2}(c)$ &.$\approx 05.56$ & -102.78 & -193.8 & -96.9 \\
\hline $5=z_{2}(c)$ & -198.0 & -99.0 & -186.7 & -93.4 \\
\hline$y \equiv=1 \quad(c)$ & .97 .755 & $-97 .-55$ & -98.232 & $-98.23 \hat{z}$ \\
\hline シニン & $-9: .70$ & -9.70 & & \\
\hline$z \equiv I_{\text {z }}(c)$ & -190.0 & -95.0 & -179.3 & -89.7 \\
\hline$z=\Sigma_{3}(c)$ & -253.6 & -8.99 & & \\
\hline$=2 I_{3}(\alpha)$ & $-260 . j$ & .. 86.8 & & \\
\hline$\equiv=I_{3}(c, \alpha)$ & -257.8 & -35.9 & & \\
\hline$N \neq=I_{3}(\alpha, c)$ & $-<54.3$ & -84.8 & & \\
\hline$\ddot{z} \equiv z_{3} i c j$ & -234.8 & -78.3 & & \\
\hline $\mathrm{P} \mathrm{L}_{3}$ (c) & -230.0 & -76.7 & & \\
\hline$\left.M=21_{2} \therefore\right)$ & -153.4 & -75.7 & -141.57 & -70.8 \\
\hline$s c=1_{3}$ & $-2=0.8$ & -73.5 & & \\
\hline $\mathrm{N}=I_{3}(\mathrm{c})$ & -216 & -73 & & \\
\hline$\because x=I_{4}(0)$ & -285 & -71.2 & & \\
\hline$I=I_{3}(c)$ & -213 & -71 & -195.9 & -65.6 \\
\hline$v=14(c)$ & -251.2 & $-6: .8$ & -230.0 & -57.5 \\
\hline$E==l_{2}$ (c) & $-1<2.3$ & -61.2 & & \\
\hline$A=I_{3}$ & -166.2 & $-55 . i$ & -152.2 & -50.73 \\
\hline$F=-I_{2}(c)$ & -81.5 & -40.8 & -72.2 & -36.1 \\
\hline $\mathrm{S}_{1}=1_{4}(1)$ & -153.0 &.$-3 \hat{0} .3$ & -136.9 & -34.2 \\
\hline$c=1_{4}(1)$ & -33.3 & -8.3 & -16.4 & -4.1 \\
\hline
\end{tabular}




\section{TABLE IV}

\section{Oxides}

\begin{tabular}{|c|c|c|c|c|}
\hline Esmound & $\Delta \mathrm{H}^{\circ} \mathrm{f}$ & $\Delta \mathrm{H}_{\mathrm{f}}^{\mathrm{N}} \mathrm{ron}$ & $\Delta \mathrm{F}_{\mathrm{f}}^{\circ}$ & $\Delta \mathrm{PO}_{\mathrm{f}} / \mathrm{bond}$ \\
\hline $\left.\mathrm{Y}_{2} \mathrm{O}_{3}:{ } \mathrm{C}\right)$ & $\because \div i+j$ & (Dpticaim) & & \\
\hline $\mathrm{Iaa}_{\mathrm{c}} \mathrm{O}_{3}(\mathrm{c})$ & $\rightarrow \equiv \dot{\theta}$ & -76.5 & & \\
\hline (ao ic) & $-i 51.9$ & -75.95 & -144.4 & -72.2 \\
\hline $\mathrm{Pr}_{2} \mathrm{O}_{3}(\mathrm{C})$ & $-4+4.5$ & -7.68 & & \\
\hline $\mathrm{Na}_{2} \mathrm{O}_{3} \quad i$ & -44200 & -7. & & \\
\hline$B \in C \quad(c)$ & $\therefore+6.0$ & $-\because 3.0$ & -139.0 & -509.5 \\
\hline Ir.J $\partial_{c}(c)$ & $-29 ?$. & -7.00 & & \\
\hline $\mathrm{MgO}(\mathrm{c})$ & -143.84 & -71.92 & -136.13 & -58.6 \\
\hline $\left.\mathrm{i1}_{2} \mathrm{O}: \vdots\right)$ & $-14 c .4$ & $-\because 1+02$ & & \\
\hline $\begin{array}{ll}\mathrm{SnO}^{\prime}(i) \\
\mathrm{PuO} \\
\mathrm{JO}_{2}(\mathrm{c})\end{array}$ & $\begin{array}{r}-141.1 \\
135 \pm 6 \\
-70 .\end{array}$ & $\begin{array}{c}-0.55 \\
68 \pm 3 \\
-6 i .5\end{array}$ & $\begin{array}{l}-133.8 \\
-257 .\end{array}$ & 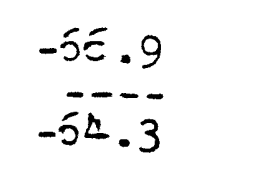 \\
\hline$B 30$ ic) & -13304 & $-66 .:$ & -126.3 & -63.2 \\
\hline $\mathrm{Al}_{2} \mathrm{C}_{3} \quad(\mathrm{C}, \alpha)$ & -399.09 & $-66 \cdot 5=$ & -376.77 & -5́2.795 \\
\hline $\mathrm{PuO}_{2}(\mathrm{c})$ & -251 & $-6 c .8$ & & \\
\hline$c \in C=(c)$ & $-\varepsilon \overline{3} 3$. &.-58.3 & & \\
\hline $\mathrm{S}_{2}$ (c) quartz & -205.4 & -51.4 & -192.4 & $-4 \varepsilon .1$ \\
\hline $\mathrm{Na}_{2} \mathrm{O}(\mathrm{c})$ & -99.4 & -49.7 & & \\
\hline$K=0 \quad(0)$ & -86.4 & -43.2 & & \\
\hline$F e_{0.95} 0$ & -63.7 & -31.9 & -58.4 & -29.2 \\
\hline Co $(\mathrm{g})$ & $-26.415 ?$ & -13.208 & -32.8079 & -16.404 \\
\hline
\end{tabular}




\section{TABLE V}

\section{Sulfides}

\begin{tabular}{|c|c|c|c|c|}
\hline Compound & $\Delta \mathrm{H}_{\mathrm{f}}^{\circ}$ & $\Delta \mathrm{H}^{\circ}{ }_{\mathrm{f}} / \mathrm{S}$ & $\Delta F^{\circ}{ }_{f}$ & $\Delta \mathrm{F}_{\mathrm{f}}^{0} / \mathrm{s}$ \\
\hline $\operatorname{cas}(\mathrm{c})$ & -115.3 & $-57 \cdot 7$ & -114.1 & -57.1 \\
\hline SrS (c) & -108.1 & 54.1 & & \\
\hline $\mathrm{BaS}(\mathrm{c})$ & -106.0 & 53.0 & & \\
\hline $\mathrm{La}_{2} \mathrm{~S}_{3}(\mathrm{c})$ & -306.8 & .51 .1 & & \\
\hline $\mathrm{K}_{2} \mathrm{~S} \quad(\mathrm{c})$ & -100 & -50 & & \\
\hline $\mathrm{Ce}_{2} \mathrm{~S}_{3}(c)$ & -298.7 & -49.8 & & \\
\hline $\mathrm{Nd}_{2} \mathrm{~S}_{3}(\mathrm{c})$ & -281.8 & -46.97 & & \\
\hline $\mathrm{Na}_{2} \mathrm{~S}^{\prime}(\mathrm{c})$ & -89.2 & -44.6 & & \\
\hline $\mathrm{Th}_{2} \mathrm{~S}_{3}$ (c) & -262.0 & -43.7 & & \\
\hline $\operatorname{MgS}(c)$ & -83.0 & -41.5 & & \\
\hline $\operatorname{BeS}(c)$ & -55.9 & -27.95 & & \\
\hline $\mathrm{Al}_{2} \mathrm{~S}_{3}(\mathrm{c})$ & -121.6 & -60.27 & -117.7 & -19.6 \\
\hline $\mathrm{S}_{1} \mathrm{~S}_{2}(\mathrm{c})$ & -34.7 & -17.4 & & \\
\hline Fes $(\alpha)$ & -22.72 & -11.36 & -23.32 & -11.66 \\
\hline $\operatorname{cs}_{2}(11 q)$ & +21.0 & +10.5 & +15.2 & +7.6 \\
\hline
\end{tabular}




\section{TABLE VI}

\section{Electromotive Series of the Elements}

L1

$\mathrm{Rb}$

$\mathrm{K}$

Ba

Sr

$\mathrm{Ca}$

$\mathrm{Na}$

$\mathrm{Mg}$

$\mathrm{Be}$

$\mathrm{U}^{+++}\left(4 \mathrm{~F}_{\mathrm{f}}=-124.4\right)$

$\mathrm{U}^{++++}\left(\Delta \mathrm{F}^{\circ}{ }_{\mathrm{f}^{\mathrm{aq}}}=-138.4\right)$

$\mathrm{Al}^{+++}\left(\Delta \mathrm{F}^{\circ} \mathrm{aq}=-125.0\right)$

$\mathrm{Mn}$

$\mathrm{Zn}$

$\mathrm{Cr}$

F'e

Cd

Co

N1

Sn

$\mathrm{Pb}$

H

$\left\{\begin{array}{l}\mathrm{Sb} \\ \mathrm{As} \\ \mathrm{B} 1\end{array}\right.$

Ag

$\mathrm{Cu}$

$\mathrm{Pd}$

$\mathrm{Hg}$

Pt

$\mathrm{Au}$ 

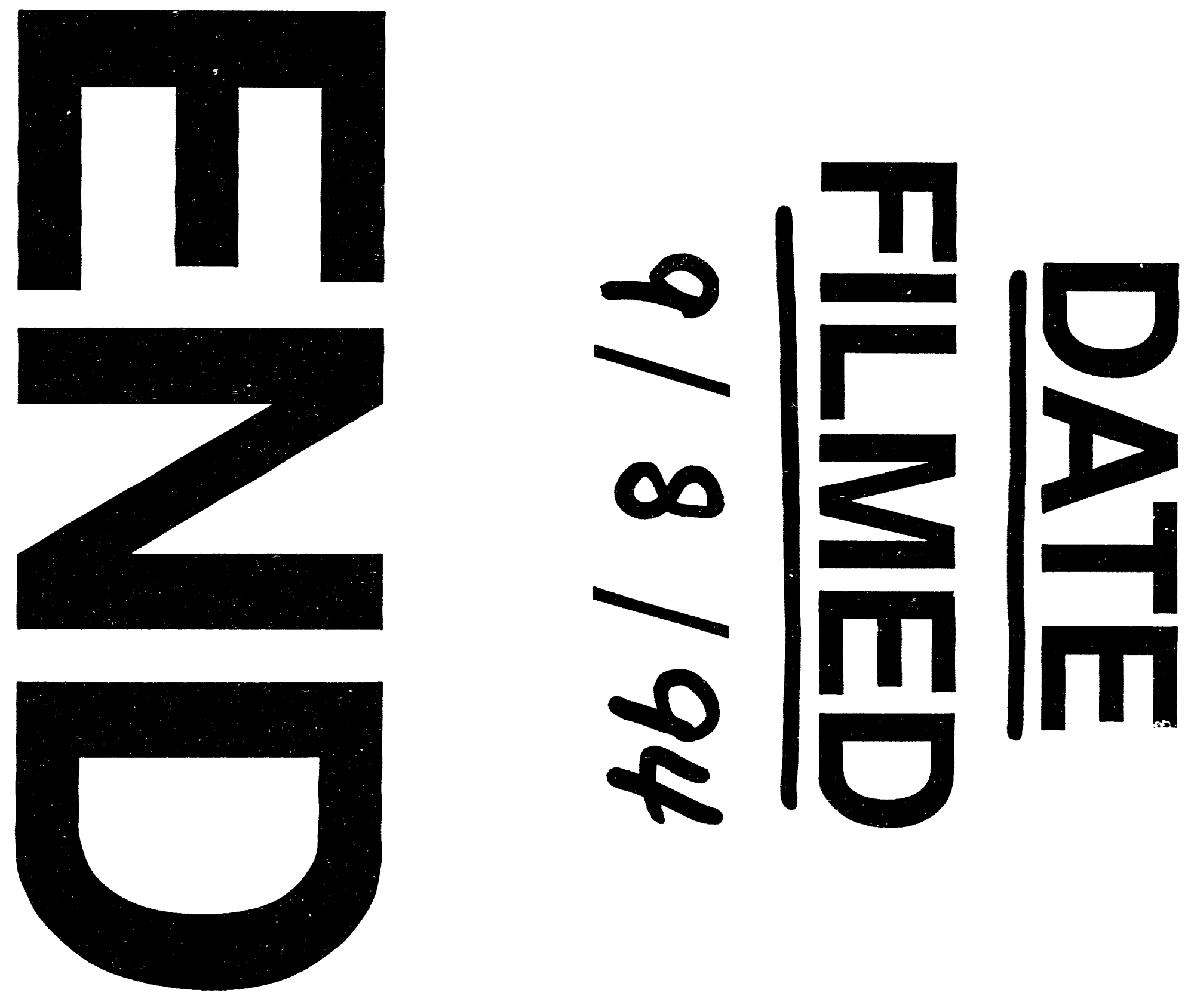